\title{
ON THE STATIC SOLUTIONS OF THE SPHERICALLY SYMMETRIC VLASOV-EINSTEIN-MAXWELL SYSTEM FOR LOW CHARGE AND ANISOTROPIC PRESSURE*
}

\author{
P. NOUNDJEU ${ }^{\dagger}$ AND G. CHENDJOU $\ddagger$
}

\begin{abstract}
We consider the Vlasov-Einstein-Maxwell (VEM) system in the spherically symmetric setting and we try to establish a global static solution with isotropic or anisotropic pressure that approaches Minkowski spacetime at the spacial infinity and has a regular center. This work extends the previous one recently done by the first author, in which only the isotropic case is concerned.
\end{abstract}

Key words. Anisotropic pressure, Newtonian limit, singularity-free solutions, ADM mass.

MSC2000. 83C20, 83C22, 34B05.

\section{Introduction}

A spacetime is said to be stationary if there is a time-like Killing vector field $v=\left(v^{\alpha}\right)$. If in addition the equation $v_{[\alpha} \nabla_{\left.\beta v_{\gamma}\right]}=0$ holds then the spacetime is static. On the one hand, from a physical point of view, static spacetimes are known to describe some phenomena in our universe. For instance it is well known that in the region $r>2 m$ where $\frac{\partial}{\partial t}$ is time-like, the Schwarzschild metric defined a Black Hole whose mass is equal to the parameter $m>0$ [16]. On the other hand, from a mathematical point of view, researching statics spacetimes may give rise to a new interesting subject to be studied using an analytical argument.

In [10], the author looked for a static global solution of the spherically symmetric VEM system for the particles with small charge $q$. We notice that in our context, particles could be those inside a relativistic charged plasma. Using $q$ as a parameter, the author cited above established a global existence theorem by means of a perturbation method. This work is concerned only with the isotropic pressure case. We now consider the general situation where the pressure depends on direction and we wish to extend the results obtained in [10] to the new context which could include the politropic solutions of the Vlasov-Poisson (VP) system.

Next, with the assumption of spherical symmetry the electromagnetic field $F$ reduces to its electric part, $E^{i}(x):=\varepsilon(r) \frac{x^{i}}{r}$, with $r$ being the Euclidean norm of the spacial vector $x$, and the VEM yields

$$
\begin{gathered}
\frac{v}{\sqrt{1+v^{2}}} \cdot \frac{\partial f}{\partial x}-\left(\mu^{\prime} \sqrt{1+v^{2}}-q e^{2 \lambda} \varepsilon\right) \frac{x}{r} \cdot \frac{\partial f}{\partial v}=0 \\
e^{-2 \lambda}\left(2 r \lambda^{\prime}-1\right)+1=8 \pi r^{2} \rho \\
e^{-2 \lambda}\left(2 r \mu^{\prime}+1\right)-1=8 \pi r^{2} p \\
\frac{d}{d r}\left(r^{2} e^{\lambda} \varepsilon\right)=q r^{2} e^{\lambda} M
\end{gathered}
$$

*Received: May 9, 2013; accepted (in revised form): September 3, 2013. Communicated by Francois Bouchut.

${ }^{\dagger}$ Department of Mathematics, Faculty of Science, University of Yaounde 1, PO Box 812, Yaounde, Cameroun (pnoundjeu@ymail.com).

${ }^{\ddagger}$ Department of Mathematics, Faculty of Science, University of Yaounde 1, PO Box 812, Yaounde, Cameroun (gchendjou@gmail.com). 
where, $x, v \in \mathbb{R}^{3}, r=|x|, \lambda^{\prime}=\frac{d \lambda}{d r}$, and

$$
\begin{aligned}
\rho(x) & =\rho(r)=\int_{\mathbb{R}^{3}} f(x, v) \sqrt{1+v^{2}} d v+\frac{1}{2} e^{2 \lambda(x)} \varepsilon^{2}(x), \\
p(x) & =p(r)=\int_{\mathbb{R}^{3}}\left(\frac{x \cdot v}{r}\right)^{2} f(x, v) \frac{d v}{\sqrt{1+v^{2}}}-\frac{1}{2} e^{2 \lambda(x)} \varepsilon^{2}(x), \\
M(x) & =M(r)=\int_{\mathbb{R}^{3}} f(x, v) d v .
\end{aligned}
$$

In the above, (1.1) is the Vlasov equation, (1.2) and (1.3) are a part of the Einstein equations, while (1.4) is a part of the Maxwell system. Notice that in the Vlasov equation, $v \cdot \frac{\partial f}{\partial x}=\sum_{i=1}^{3} v^{i} \frac{\partial f}{\partial x^{i}}, q$ denotes the charge of particles, and $\lambda$ and $\mu$ denote the metric functions. Here $f$ is spherically symmetric if $f(A x, A v)=f(x, v)$, for $x, v \in \mathbb{R}^{3}$, $A \in S O(3)$. We also recall that $\rho$ stands for the energy density of the system while $p$ represents the radial pressure. The spacetime we are looking for is $\mathbb{R}^{4}$, endowed with the metric that is invariant under rotations:

$$
d s^{2}=-e^{2 \mu} d t^{2}+e^{2 \lambda} d r^{2}+r^{2}\left(d \theta^{2}+\sin ^{2} \theta d \varphi^{2}\right),
$$

in which $t \in \mathbb{R}, r \geq 0, \theta \in[0, \pi]$, and $\varphi \in[0,2 \pi]$. We are also looking for the asymptotically flat solutions with a regular center that allow us to prescribe the following boundary conditions:

$$
\lim _{r \rightarrow+\infty} \lambda(r)=\lim _{r \rightarrow+\infty} \mu(r)=\lambda(0)=0 .
$$

Again for the regularity of $\varepsilon$, we will need the following additional boundary conditions:

$$
\lim _{r \rightarrow+\infty} \varepsilon(r)=\varepsilon(0)=0 .
$$

We encourage the reader to obtain more details on how to establish the above equations in [11].

Next, in the related literature, the initial value problem for the corresponding time dependent is investigated in [11]. Again the Newtonian limit of the spherically symmetric VEM is discussed in [12] and this work extends the one of Rein in [15]. Moreover, in [18] the authors prove the existence of a globally defined smooth static solution for the Einstein-Yang-Mills equations with $S U(2)$ gauge group. Also, global static solutions are established in [1] for the VP system. We also notice that cylindrically symmetric, static solutions have been found by J. Batt in [5] for the Vlasov-Poisson (VP) System. A construction of global static solutions by numerical means has been made by H. Andréasson in [3] for the spherically symmetric VEM system and the same kind of solutions were found by Alan Parry in [2] for the Einstein-Klein-Gordon system.

Now, why our problem is interesting? Firstly, as we said before the choice of the ansatz for the distribution function $f$ includes the polytropic case, and the sources terms of our system seem to be more complicated than those obtained in the istropic case. Also, the proof of the global existence theorem seems to be simpler than that of the tropic case, because the boundary conditions on our solutions force the source terms of the VEM system to be bounded and, using a generalized form of the Gronwall 
lemma, one extends a local solution to a global one. Secondly, to prove the asymptotic flatness of our spacetime we need to prove Theorem 4.1, from which the finiteness of the ADM mass is deduced. This fact is not important in the isotropic setting because the exterior region can be covered by the Reissner-Norsdtöm solution.

The present work proceeds as follows: In Section 2, considering $f$ as function of the local energy $E$ and the square of angular momentum $L$, we write down the corresponding sources terms of the fields equations and then we obtain the reduced system. In Section 3, we try to prove the existence of solutions while in Section 4, we show the finiteness of the ADM mass that allows us to conclude the asymptotic flatness of our spacetime. We summarize this work in Section 5.

\section{Conserved quantities and reduction of the problem}

We aim to express the full system as a nonlinear integrodifferential system for $\lambda, \mu$, and $\varepsilon$. Now, the characteristic system that corresponds to the Vlasov equation yields

$$
\begin{aligned}
\dot{x} & =\frac{v}{1+v^{2}}, \\
\dot{v} & =-\left(\mu^{\prime} \sqrt{1+v^{2}}-q e^{2 \lambda} \varepsilon\right) \frac{x}{r} .
\end{aligned}
$$

We will use the following result when establishing our reduced system.

Lemma 2.1. If $f$ is spherically symmetric, then for all $(x, v) \in \mathbb{R}^{6}$, with $x \neq 0$ and $|\omega|<u$, where $\omega=\frac{x \cdot v}{r}$, one can write $f(x, v)=f(r, u, \alpha)$, with $u=|v|$ and $\cos \alpha=\frac{x \cdot v}{r u}$, $\alpha \in[0, \pi]$.

Next, a straightforward calculation shows that the quantities

$$
E:=e^{\mu(r)} \sqrt{1+v^{2}}-q \int_{0}^{r} e^{\mu+2 \lambda} \varepsilon d s ; \quad L:=r^{2} v^{2}-(x . v)^{2}
$$

are conserved along the characteristics. We recall that $E$ is the particle energy [1] and $\sqrt{L}$ is modulus of the angular momentum. We now set $f(x, v)=\Phi(E, L)$, for a fixed function $\Phi$. Then, $f$ satisfies the Vlasov equation and we can write, using the polar coordinates $r=|x|, u=|v|, v^{1}=u \cos \varphi \sin \alpha, v^{2}=u \sin \varphi \sin \alpha, v^{3}=u \cos \alpha, \alpha \in[0, \pi]$, $\varphi \in[0,2 \pi]$ with $\cos \alpha=\frac{x \cdot v}{r u}$, and using Lemma 2.1,

$$
\begin{aligned}
\rho(r) & =\frac{2 \pi}{r^{2}} \int_{1}^{+\infty} \int_{0}^{r^{2}\left(\tau^{2}-1\right)} \frac{\tau^{2}}{\sqrt{\tau^{2}-1-L / r^{2}}} \Phi(E, L) d L d \tau+\frac{1}{2} e^{2 \lambda(r)} \varepsilon^{2}(r), \\
p(r) & =\frac{2 \pi}{r^{2}} \int_{1}^{+\infty} \int_{0}^{r^{2}\left(\tau^{2}-1\right)} \Phi(E, L) \sqrt{\tau^{2}-1-L / r^{2}} d L d \tau-\frac{1}{2} e^{2 \lambda(r)} \varepsilon^{2}(r), \\
M(r) & =\frac{2 \pi}{r^{2}} \int_{1}^{+\infty} \int_{0}^{r^{2}\left(\tau^{2}-1\right)} \frac{\tau}{\sqrt{\tau^{2}-1-L / r^{2}}} \Phi(E, L) d L d \tau .
\end{aligned}
$$

We are looking for solutions with an anisotropic pressure, which allows us to require $\Phi$ to satisfy the following.

\section{Assumption on $\Phi$.}

$$
\Phi(E, L)=\Phi(E) L^{l}, E>0, L>0,
$$

with $l>-1 / 2$, and $\Phi \in L^{\infty}(] 0,+\infty[)$ is nonnegative and compactly supported. 
REMARK 2.2. We notice that the form of $\Phi(E, L)$ depends on the physical models that are studied. For example if one wishes to study the Wooley-Dickens models or the Truncated Maxwell-Boltzmann models then it will be convenient to set $\Phi(E, L)=$ $e^{E_{0}-E}$ for $E<E_{0}$ and zero otherwise. For the polytropic solutions of the VlasovPoisson system one usually takes $\Phi(E, L)=\left(E_{0}-E\right)^{k} L^{l}$ for $E<E_{0}$ and zero otherwise. The reader can refer to J. Binney in [7], S.L. Shapiro in [17], and J. Batt in [6] to obtain more details on the static spherically symmetric models.

Once again, $f(x, v)=\Phi(E) L^{l}$ defines a solution of the Vlasov equation and we obtain, by the change of variables $L^{\prime}=\frac{L}{r^{2}\left(\tau^{2}-1\right)}$,

$\rho(r)=g_{\Phi}(r, \mu(r), \lambda(r), \varepsilon(r)) ; p(r)=h_{\Phi}(r, \mu(r), \lambda(r), \varepsilon(r)) ; M(r)=l_{\Phi}(r, \mu(r), \lambda(r), \varepsilon(r))$, where

$$
\begin{gathered}
g_{\Phi}(r, u, v, w):=2 \pi C_{l,-1 / 2} r^{2 l} \int_{1}^{+\infty} \tau^{2} \Phi(E)\left(\tau^{2}-1\right)^{l+1 / 2} d \tau+\frac{1}{2} e^{2 v} w^{2}, \\
h_{\Phi}(r, u, v, w):=2 \pi C_{l, 1 / 2} r^{2 l} \int_{1}^{+\infty} \Phi(E)\left(\tau^{2}-1\right)^{l+3 / 2} d \tau-\frac{1}{2} e^{2 v} w^{2}, \\
l_{\Phi}(r, u, v, w):=2 \pi C_{l,-1 / 2} r^{2 l} \int_{1}^{+\infty} \tau \Phi(E)\left(\tau^{2}-1\right)^{l+1 / 2} d \tau,
\end{gathered}
$$

where in the above equations we set $C_{a, b}=\int_{0}^{1} s^{a}(1-s)^{b} d s, a>-1, b>-1$. Before continuing our investigation, we give details on how to establish for instance the expression given by (2.1). Once this is done the reader could apply the same method to establish (2.2) and (2.3). We will focus on the first term on the right hand side of $\rho(r)$, that is denoted by $A$. So in this expression we take $\Phi(E, L)=\Phi(E) L^{l}$ and we can write

$$
\begin{aligned}
A & =\frac{2 \pi}{r^{2}} \int_{1}^{+\infty} \tau^{2} \Phi(E) d \tau \int_{0}^{r^{2}\left(\tau^{2}-1\right)} \frac{L^{l} d L}{\sqrt{\tau^{2}-1-L / r^{2}}} \\
& =2 \pi C_{l,-1 / 2} r^{2 l} \int_{1}^{+\infty} \tau^{2} \Phi(E)\left(\tau^{2}-1\right)^{l+1 / 2} d \tau,
\end{aligned}
$$

where we made the change of the variable $L^{\prime}=\frac{L}{r^{2}\left(\tau^{2}-1\right)}$, and (2.1) is deduced. We also set $E=\tau e^{\mu(r)}+j(\mu(r), \lambda(r), \varepsilon(r))$ with

$$
j(\mu(r), \lambda(r), \varepsilon(r)):=-q \int_{0}^{r} e^{\mu+2 \lambda} \varepsilon d s .
$$

So, the VEM system reduces to the following equations:

$$
\begin{gathered}
e^{-2 \lambda}\left(2 r \lambda^{\prime}-1\right)+1=8 \pi r^{2} g_{\Phi}(r, \mu, \lambda, \varepsilon), \\
e^{-2 \lambda}\left(2 r \mu^{\prime}+1\right)-1=8 \pi r^{2} h_{\Phi}(r, \mu, \lambda, \varepsilon), \\
\frac{d}{d r}\left(r^{2} e^{\lambda} \varepsilon\right)=q r^{2} e^{\lambda} l_{\Phi}(r, \mu, \lambda, \varepsilon) .
\end{gathered}
$$


The integration of $(2.4)$ on $[0, r]$ with $\lambda(0)=0$ yields

$$
e^{-2 \lambda}=1-\frac{8 \pi}{r} \int_{0}^{r} s^{2} g_{\Phi}(s, \mu(s), \lambda(s), \varepsilon(s)) d s,
$$

and, inserting this in (2.5), one has

$$
\begin{aligned}
\mu^{\prime}(r)= & \frac{4 \pi r h_{\Phi}(r, \mu(r), \lambda(r), \varepsilon(r))}{1-\frac{8 \pi}{r} \int_{0}^{r} s^{2} g_{\Phi}(s, \mu(s), \lambda(s), \varepsilon(s)) d s} \\
& \quad+\frac{4 \pi \int_{0}^{r} s^{2} g_{\Phi}(s, \mu(s), \lambda(s), \varepsilon(s)) d s}{r^{2}\left(1-\frac{8 \pi}{r} \int_{0}^{r} s^{2} g_{\Phi}(s, \mu(s), \lambda(s), \varepsilon(s)) d s\right)} .
\end{aligned}
$$

Next (2.6) yields, by the integration on $[0, r]$,

$$
\varepsilon(r)=\frac{q}{r^{2}} e^{-\lambda} \int_{0}^{r} e^{\lambda(s)} s^{2} l_{\Phi}(s, \mu(s), \lambda(s), \varepsilon(s)) d s .
$$

In the sequel, we try to solve the reduced system (2.7)-(2.9) globally on $[0,+\infty[$.

\section{Existence of solutions}

First of all we show that for functions $\Phi$ that satisfy the above assumption, the functions $g_{\Phi}, h_{\Phi}$, and $l_{\Phi}$ are $C^{1}$. This will allow us to conclude that a solution of our reduced system will be a regular one.

Lemma 3.1. Let $\Phi \in L^{\infty}(] 0,+\infty[)$ be a compactly supported function. Then the functions defined by (2.1)-(2.3) belong to $C^{1}\left(\mathbb{R}^{4}\right)$.

Proof. Let $\Phi$ be as above. Using the compactness of $\Phi$, these functions are well defined. Besides, with the help of the change of variables $E=\tau e^{\mu}+j(r)$, where the function $j$ is given by $\left(2.3^{\prime}\right)$, one obtains

$$
\begin{aligned}
& g_{\Phi}(r, u, v, w)=2 \pi C_{l,-1 / 2} r^{2 l} e^{-(2 l+4) u} \tilde{g}\left(u, e^{u}+j(u, v, w)\right)+\frac{1}{2} e^{2 v} w^{2}, \\
& h_{\Phi}(r, u, v, w)=2 \pi C_{l, 1 / 2} r^{2 l} e^{-(2 l+4) u} \tilde{h}\left(u, e^{u}+j(u, v, w)\right)-\frac{1}{2} e^{2 v} w^{2}, \\
& l_{\Phi}(r, u, v, w)=2 \pi C_{l,-1 / 2} r^{2 l} e^{-(2 l+3) u} \tilde{l}\left(u, e^{u}+j(u, v, w)\right),
\end{aligned}
$$

where

$$
\begin{aligned}
& \tilde{g}(u, t)=\int_{t}^{+\infty} \Phi(E)\left(E+e^{u}-t\right)^{2}\left[\left(E+e^{u}-t\right)^{2}-e^{2 u}\right]^{l+1 / 2} d E, \\
& \tilde{h}(u, t)=\int_{t}^{+\infty} \Phi(E)\left[\left(E+e^{u}-t\right)^{2}-e^{2 u}\right]^{l+3 / 2} d E \\
& \tilde{l}(u, t)=\int_{t}^{+\infty} \Phi(E)\left(E+e^{u}-t\right)\left[\left(E+e^{u}-t\right)^{2}-e^{2 u}\right]^{l+1 / 2} d E,
\end{aligned}
$$

with $t>0$ and $j \in C^{1}\left(\mathbb{R}^{3}\right.$ ) (deduced from (2.3')), replacing $\mu, \lambda$ and $\varepsilon$ by $u, v$, and $w$ respectively. We now prove that the function $\tilde{g}, \tilde{h}$, and $\tilde{l}$ are $C^{1}$ on $\mathbb{R}^{2}$ and with this we can conclude that the same property holds for $g_{\Phi}, h_{\Phi}$, and $l_{\Phi}$ on $\mathbb{R}^{4}$. Next for $t>0, \Delta t>0$ such that $t-\Delta t>0$, one has, for

$$
A:=\frac{1}{\Delta t}(\tilde{h}(u, t-\Delta t)-\tilde{h}(u, t))
$$




$$
\begin{aligned}
A= & \frac{1}{\Delta t} \int_{t-\Delta t}^{t} \Phi(E)\left[\left(E+e^{u}-t+\Delta t\right)^{2}-e^{2 u}\right]^{l+3 / 2} d E \\
& \quad+\int_{t}^{+\infty} \frac{\Phi(E)}{\Delta t}\left\{\left[\left(E+e^{u}-t+\Delta t\right)^{2}-e^{2 u}\right]^{l+3 / 2}-\left[\left(E+e^{u}-t\right)^{2}-e^{2 u}\right]^{l+3 / 2}\right\} d E \\
:=I_{1}+I_{2} . &
\end{aligned}
$$

Using the mean value theorem, one observes that $\lim _{\Delta t \rightarrow 0^{+}} I_{1}=0$. On the one hand, using Lebesgue's dominated convergence theorem, one concludes that $\lim _{\Delta t \rightarrow 0^{+}} I_{2}$ exists and the left derivative function of $\tilde{h}_{u}$ is in the form

$$
\begin{aligned}
\frac{\partial \tilde{h}}{\partial t}(u, t) & =(2 l+3) \int_{t}^{+\infty} \Phi(E)\left(E+e^{u}-t\right)\left[\left(E+e^{u}-t\right)^{2}-e^{2 u}\right]^{l+1 / 2} d E \\
& =(2 l+3) \tilde{l}(u, t) .
\end{aligned}
$$

Thus $\tilde{h}$ has a partial derivative with respect to $t$ on $\mathbb{R}_{+}$and, using once again the Lebesgue dominated convergence theorem, this partial derivative is continuous. Besides, using again the compactness of $\Phi$ and the Lebesgue's theorem, one deduces that $\tilde{h}$ has a partial derivative with respect to $u$ which is continuous, that is

$$
\frac{\partial \tilde{h}}{\partial u}(u, t)=(2 l+3) e^{u} \tilde{l}(u, t)-(2 l+3) e^{2 u} \bar{g}(u, t)
$$

where

$$
\bar{g}(u, t):=\int_{t}^{+\infty} \Phi(E)\left[\left(E+e^{u}-t\right)^{2}-e^{2 u}\right]^{l+1 / 2} d E,
$$

and because $\bar{g}$ is $C^{1}$, so is the function $\tilde{h}$ and one can proceed as above to obtain the same result for both functions $\tilde{g}$ and $\tilde{l}$.

Next we state the local existence and uniqueness of $\lambda, \mu$, and $\varepsilon$, whose proof is the same as the one of Theorem 3.2 in [10].

TheOREM 3.2 (Local existence). Let $\Phi$ be as described in the general assumption and let $g_{\Phi}, h_{\Phi}$, and $l_{\Phi}$ be defined by (2.1)-(2.3). Take the charge $q$ of particles small so that the local energy $E$ is nonnegative. Then for every $r_{0} \geq 0$ and $\lambda_{0}, \mu_{0}, \varepsilon_{0} \in \mathbb{R}$ with $\lambda_{0}=\varepsilon_{0}=0$ if $r_{0}=0$, there exists a unique maximal solution $\lambda, \mu \in C^{2}\left(\left[r_{0}, R[)\right.\right.$, $\varepsilon \in C^{1}\left(\left[r_{0}, R[)\right.\right.$ of system (2.4)-(2.6) with $\lambda\left(r_{0}\right)=\lambda_{0}, \mu\left(r_{0}\right)=\mu_{0}$, and $\varepsilon\left(r_{0}\right)=\varepsilon_{0}$.

In order to show that the solutions above extend to a global one we will use the following generalization of the Gronwall inequality, whose proof can be found in [9].

Lemma 3.3. Let $F=F(t, u)$ be a real-valued function on $\mathbb{R}^{2}$ such that $F$ and $\frac{\partial F}{\partial u}$ are continuous. Let $U$ and $V$ be a real-valued functions that satisfy

$$
\begin{gathered}
U^{\prime}(t)=F(t, U(t)), \quad U(0)=A, \\
V(t) \leq A+\int_{0}^{t} F(s, V(s)) d s .
\end{gathered}
$$

Then, for all $t \geq 0, V(t) \leq U(t)$, provided $\frac{\partial F}{\partial u} \geq 0$.

We now state the global existence theorem for our system. 
TheOREm 3.4 (Global existence). Let $\Phi$ be as stated in the general assumption. Let $g_{\Phi}, h_{\Phi}$, and $l_{\Phi}$ be defined by (2.1), (2.2), and (2.3). Take $r_{0} \geq 0, \lambda_{0} \geq 0, \varepsilon_{0} \geq 0$, $\mu_{0} \leq 0$ with $\lambda_{0}=\varepsilon(0)=0$ if $r_{0}=0$. Then for $q$ small enough, there exists a unique solution $\mu, \lambda \in C^{2}\left(\left[r_{0},+\infty[)\right.\right.$ and $\varepsilon \in C^{1}\left(\left[r_{0},+\infty[)\right.\right.$ of the system (2.4)-(2.6) that satisfies $\mu\left(r_{0}\right)=\mu_{0}, \lambda\left(r_{0}\right)=\lambda_{0}$, and $\varepsilon\left(r_{0}\right)=\varepsilon_{0}$.

Proof. Let $\mu, \lambda$, and $\varepsilon$ be a regular solution of the system (2.4)-(2.6) on the right maximal interval $\left[r_{0}, R[\right.$. The existence of this solution is ensured by Theorem 3.2. Next, one has the equations (1.2), (1.3), and (1.4) on $\left[r_{0}, R[\right.$. We now assume that $R<+\infty$. Then for $r_{0}>0,(1.2)$ yields by integration on $\left[r_{0}, r\right]$,

$$
\lambda(r)=\lambda_{0}+\frac{1}{2} \int_{r_{0}}^{r} \frac{d s}{s}+\frac{1}{2} \int_{r_{0}}^{r} \frac{e^{2 \lambda(s)}\left(-1+8 \pi s^{2} \rho(s)\right)}{s} d s,
$$

and using the fact that $\lambda \geq 0$, one obtains by the Hölder inequality and having in mind that the boundary conditions force the fixed functions $g_{\Phi}, h_{\Phi}$, and $l_{\Phi}$ to have finite $L^{\infty}$ norm,

$$
\lambda(r) \leq C+C\left(\left\|g_{\Phi}\right\|_{L^{\infty}}\right) \int_{r_{0}}^{r} e^{2 \lambda(s)} d s,
$$

and applying Lemma 3.3, for $F(r, u)=C e^{2 u}, V(r)=\lambda(r)$, and

$$
U(r)=\frac{-1}{2} \log \left(e^{-2 U\left(r_{0}\right)}-2 C\left(r-r_{0}\right)\right),
$$

one obtains that, because $U$ is increasing,

$$
\lambda(r) \leq U(r) \leq U(R) \leq C, \quad r \in\left[r_{0}, R[.\right.
$$

Besides, using the fact that $\lambda$ is bounded on $\left[r_{0}, R[\right.$, equation (1.3) shows that the same property holds for $\mu$. Again, because $\lambda \geq 0$, one also deduces from equation (1.4) that $\varepsilon$ is bounded on $\left[r_{0}, R\left[\right.\right.$. Moreover we deduce from (2.4) and (2.5) that $\lambda^{\prime}$ and $\mu^{\prime}$ are bounded on $\left[r_{0}, R[\right.$. So our solution $(\mu, \lambda, \varepsilon)$ lies on a compact set $K$ of $\mathbb{R}^{3}$ and applying Theorem 3, Section 2.4 of [8], we conclude that $R=+\infty$, which is a contradiction. Thus the proof of Theorem 3.4 is complete.

REMARK 3.5. In the anisotropic case (i.e $\left.f(x, v)=\Phi(E) L^{l}\right)$, the regularity of $f$ depends on that of $\Phi$. So, for instance if $\Phi$ is a $C^{1}$ function, then $f$ will be a $C^{1}$ one too. Thus, $(\Phi(E), \lambda, \mu, \epsilon)$ is a regular solution of the full EVM system. We also notice that the proof of the global existence theorem in this paper is simpler than the one of $[10]$.

\section{Singularity-free solutions with finite mass and finite radius}

We are interested in smooth, singularity-free solutions with a regular center and finite mass. These properties hold for solutions of the VP system. So, we will conclude the same for our case if the Newtonian limit of the VEM system is the VP system. To do so, the metric defined over the space-like hypersurface $\left\{t=t_{0}\right\}$ is rescaling by $\gamma=\frac{1}{c^{2}}$, where $c$ denotes the speed of light and one has to prove, as in the uncharged case, that the corresponding $\mathrm{VEM}_{\gamma}$ converges to the $\mathrm{VP}$ system as $\gamma$ goes to 0 . Let $\nu:=\frac{1}{\gamma} \mu \cdot$ We deduce from [12] that the $\mathrm{VEM}_{\gamma}$ yields

$$
\frac{v}{\sqrt{1+\gamma v^{2}}} \cdot \frac{\partial f}{\partial x}-\left(\nu^{\prime} \sqrt{1+\gamma v^{2}}-q e^{2 \lambda} \varepsilon\right) \frac{x}{r} \cdot \frac{\partial f}{\partial v}=0
$$




$$
\begin{gathered}
e^{-2 \lambda}\left(2 r \lambda^{\prime}-1\right)+1=8 \pi \gamma r^{2} \rho, \\
e^{-2 \lambda}\left(2 r \nu^{\prime}+\frac{1}{\gamma}\right)-\frac{1}{\gamma}=8 \pi \gamma r^{2} p, \\
\frac{d}{d r}\left(r^{2} e^{\lambda} \varepsilon\right)=q r^{2} e^{\lambda} M,
\end{gathered}
$$

where

$$
\begin{gathered}
\rho(x)=\int_{\mathbb{R}^{3}} \sqrt{1+\gamma v^{2}} f(x, v) d v+\frac{\gamma}{2} e^{2 \lambda(x)} \varepsilon^{2}(x), \\
p(x)=\int_{\mathbb{R}^{3}}\left(\frac{x \cdot v}{r}\right)^{2} f(x, v) \frac{d v}{\sqrt{1+\gamma v^{2}}}-\frac{1}{2} e^{2 \lambda(x)} \varepsilon^{2}(x),
\end{gathered}
$$

and $M$ is the same as in Section 1. To obtain the right limit we take the local energy of particles in the form

$$
E=\frac{1}{\gamma}\left(e^{\gamma \nu} \sqrt{1+\gamma v^{2}}-1\right)-q \int_{0}^{r} e^{\gamma \nu+2 \lambda} \varepsilon d s
$$

and $L$ remains unchanged. As in Section 2, we consider the same ansatz for the distribution function $f$, that is $f(x, v)=\Phi(E) L^{l}$, with $\Phi \in L^{\infty}(] 0,+\infty[)$ compactly supported. Then the corresponding $\operatorname{EVM}_{\gamma}$ yields

$$
\begin{gathered}
e^{-2 \lambda}\left(2 r \lambda^{\prime}-1\right)+1=8 \pi \gamma r^{2} g_{\Phi, \gamma}(r, \nu(r), \lambda(r), \varepsilon(r)), \\
e^{-2 \lambda}\left(2 r \nu^{\prime}+\frac{1}{\gamma}\right)-\frac{1}{\gamma}=8 \pi \gamma r^{2} h_{\Phi, \gamma}(r, \nu(r), \lambda(r), \varepsilon(r)), \\
\frac{d}{d r}\left(r^{2} e^{\lambda} \varepsilon\right)=q r^{2} e^{\lambda} l_{\Phi, \gamma}(r, \nu(r), \lambda(r), \varepsilon(r)),
\end{gathered}
$$

with

$$
\begin{aligned}
g_{\Phi, \gamma}(r, \nu(r), \lambda(r), \varepsilon(r)):=2 \pi r^{2 l} C_{l,-1 / 2} e^{-\gamma(2 l+4) \nu(r)} \tilde{g}_{\Phi, \gamma}\left(\nu(r), 1 / \gamma e^{\gamma \nu(r)}-1 / \gamma+j(r)\right) & \\
& +\frac{\gamma}{2} e^{2 \lambda(r)} \varepsilon^{2}(r),
\end{aligned}
$$

We notice that in the above,

$$
j(r)=-q \int_{0}^{r} e^{\gamma \nu+2 \lambda} \varepsilon d s
$$




$$
\begin{aligned}
\tilde{g}_{\Phi, \gamma}(u, t) & :=\int_{t}^{+\infty} \Phi(E)\left(\gamma E-\gamma t+e^{\gamma u}\right)^{2}\left[\frac{1}{\gamma}\left(\gamma E-\gamma t+e^{\gamma u}\right)^{2}-\frac{1}{\gamma} e^{2 \gamma u}\right]^{l+1 / 2} d E, \\
\tilde{h}_{\Phi, \gamma}(u, t) & :=\int_{t}^{+\infty} \Phi(E)\left[\frac{1}{\gamma}\left(\gamma E-\gamma t+e^{\gamma u}\right)^{2}-\frac{1}{\gamma} e^{2 \gamma u}\right]^{l+3 / 2} d E \\
\tilde{l}_{\Phi, \gamma}(u, t) & :=\int_{t}^{+\infty} \Phi(E)\left(\gamma E-\gamma t+e^{\gamma u}\right)\left[\frac{1}{\gamma}\left(\gamma E-\gamma t+e^{\gamma u}\right)^{2}-\frac{1}{\gamma} e^{2 \gamma u}\right]^{l+1 / 2} d E .
\end{aligned}
$$

Besides, in the Newtonian case, the corresponding ansatz for $f$ is

$$
f(x, v)=\Phi\left(v^{2} / 2+U(r)+j_{N}(r)\right) L^{l},
$$

with

$$
\begin{gathered}
\left(r^{2} \varepsilon_{N}\right)^{\prime}(r)=q r^{2} M, \\
U^{\prime}(r)=\frac{4 \pi}{r^{2}} \int_{0}^{r} s^{2} M(s) d s,
\end{gathered}
$$

and

$$
M(r)=M(x)=\int_{\mathbb{R}^{3}} f(x, v) d v=2 \pi r^{2 l} C_{l,-1 / 2} g_{0}\left(U(r)+j_{N}(r)\right),
$$

where

$$
\begin{gathered}
j_{N}(r):=-q \int_{0}^{r} \varepsilon_{N}(s) d s, \\
g_{0}(t):=\int_{t}^{+\infty} \Phi(E)(2 E-2 t)^{l+1 / 2} d E .
\end{gathered}
$$

We now state the important result of this section.

THEOREM 4.1. For every $R>0$, there exists constants $C>0$ and $\gamma_{0}>0$ such that for every $\gamma \in\left[0, \gamma_{0}\right]$, the solution $\left(\nu_{\gamma}, \lambda_{\gamma}, \varepsilon_{\gamma}\right)$ of the system (4.8)-(4.10) with $\nu_{\gamma}(0)=\nu_{0}$, $\lambda_{\gamma}(0)=0$, and $\varepsilon_{\gamma}(0)=0$ satisfies the estimate

$$
\left|\lambda_{\gamma}(r)\right|,\left|\nu_{\gamma}(r)-U(r)\right|, \quad\left|\varepsilon_{\gamma}(r)-\varepsilon_{N}(r)\right| \leq C \gamma^{\min \{l+1 / 2,1\}}, \quad r \in[0, R],
$$

where $\left(U, \varepsilon_{N}\right)$ is the global solution of system (4.12)-(4.13) that takes $\left(\nu_{0}, 0\right)$ at $r=0$.

Proof. We are going to follow the techniques used in proving Theorem 4.1 of [14]. Consider the global solution $(\mu, \lambda, \varepsilon)$ of the $(E V M)_{\gamma}$ system that takes $\left(\nu_{0}, 0,0\right)$ at $r=0$. We also fix a global solution $\left(U, \varepsilon_{N}\right)$ of the VP system that passes by $\left(\nu_{0}, 0\right)$ at $r=0$. Using equation (4.2), one has by integration

$$
e^{-2 \lambda}=1-\frac{8 \pi \gamma}{r} \int_{0}^{r} s^{2} g_{\Phi, \gamma}(s, \nu(s), \lambda(s), \varepsilon(s)) d s,
$$


and inserting (4.15) in (4.3), we obtain the following equation:

$$
\begin{aligned}
\nu^{\prime}(r)= & \frac{4 \pi \gamma r^{2 l+1} h_{\Phi, \gamma}(r, \nu(r), \lambda(r), \varepsilon(r))}{1-\frac{8 \pi \gamma}{r} \int_{0}^{r} s^{2} g_{\Phi, \gamma}(s, \nu(s), \lambda(s), \varepsilon(s)) d s} \\
& +\frac{4 \pi}{1-\frac{8 \pi \gamma}{r} \int_{0}^{r} s^{2} g_{\Phi, \gamma}(s, \nu(s), \lambda(s), \varepsilon(s)) d s} \frac{1}{r^{2}} \int_{0}^{r} s^{2 l+1} g_{\Phi, \gamma}(s, \nu(s), \lambda(s), \varepsilon(s)) d s .
\end{aligned}
$$

Next, using (4.13) and (4.16), one has

$$
\left|\nu^{\prime}(r)-U^{\prime}(r)\right| \leq I_{1}+I_{2}+I_{3}+I_{4},
$$

where

$$
\begin{aligned}
I_{1} & =\frac{4 \pi \gamma r^{2 l+1} h_{\Phi, \gamma}(r, \nu(r), \lambda(r), \varepsilon(r))}{1-\frac{8 \pi \gamma}{r} \int_{0}^{r} s^{2} g_{\Phi, \gamma}(s, \nu(s), \lambda(s), \varepsilon(s)) d s}, \\
I_{2} & =\frac{4 \pi}{r^{2}}\left|\frac{1}{1-\frac{8 \pi \gamma}{r} \int_{0}^{r} s^{2} g_{\Phi, \gamma}(s, \nu(s), \lambda(s), \varepsilon(s)) d s}-1\right| \int_{0}^{r} s^{2 l+2} g_{\Phi, \gamma}(s, \nu(s), \lambda(s), \varepsilon(s)) d s, \\
I_{3} & =\frac{4 \pi}{r^{2}} \int_{0}^{r} s^{2 l+2}\left|g_{\Phi, \gamma}(s, \nu(s), \lambda(s), \varepsilon(s))-g_{0}(U(s)+j(s))\right| d s, \\
I_{4} & =\frac{4 \pi}{r^{2}} \int_{0}^{r} s^{2 l+2}\left|g_{0}(\nu(s)+j(s))-g_{0}\left(U(s)+j_{N}(s)\right)\right| d s .
\end{aligned}
$$

Because $\Phi$ is compactly supported, there is a number $E_{0}>0$ such that $\Phi(E)=0$ for $E>E_{0}$. So, we use this to obtain

$$
\tilde{g}_{\Phi, \gamma}(r, \nu(r), \lambda(r), \varepsilon(r)), \tilde{h}_{\Phi, \gamma}(r, \nu(r), \lambda(r), \varepsilon(r)), \tilde{l}_{\Phi, \gamma}(r, \nu(r), \lambda(r), \varepsilon(r)) \leq C .
$$

Besides, (4.15) yields, by derivation,

$$
\begin{aligned}
\lambda^{\prime}(r)=- & \frac{8 \pi^{2} \gamma}{r^{2}} e^{2 \lambda} \int_{0}^{r} s^{2 l+2} e^{-\gamma(2 l+4) \nu} \tilde{g}_{\Phi, \gamma}(s, \nu(s), \lambda(s), \varepsilon(s)) d s \\
& -\frac{4 \pi \gamma^{2}}{r^{2}} e^{2 \lambda} \int_{0}^{r} s^{2} e^{2 \lambda(s)} \varepsilon^{2}(s) d s \\
& +4 \pi \gamma r^{2 l+1} e^{2 \lambda} C_{l,-1 / 2} e^{-\gamma(2 l+4) \nu} \tilde{g}_{\Phi, \gamma}(r, \nu(r), \lambda(r), \varepsilon(r))+4 \pi \gamma^{2} r e^{4 \lambda} \varepsilon^{2},
\end{aligned}
$$

and using equation (4.10), the Hölder inequality, and the fact that $\lambda(0)=0$, one obtains the following estimate:

$$
\lambda(r) \leq C \gamma+C \gamma^{2} \int_{0}^{r} e^{4 \lambda(s)} d s, \gamma \in\left[0, \gamma_{0}\right]
$$

for some $\gamma_{0}$ small enough. So, we can use Lemma 3.3 in which $V(r)=\lambda(r)$ and $U(r)=-\frac{1}{4} \log \left(-4 C \gamma^{2} r+e^{-4 C \gamma}\right)$ to get, because $U$ is increasing,

$$
\lambda(r) \leq U(r) \leq U(R) \leq C \gamma,
$$

where the last inequality above is obtained from the fact that $U(R) / \gamma$ goes to $C \neq 0$ as $\gamma$ goes to 0 . Because $\lambda \geq 0$, we can obtain the same inequality for $\varepsilon$, using once again (4.10). Next, we now establish an estimate for $I_{1}$. In order to do so, we set

$$
B:=\frac{8 \pi \gamma}{r} \int_{0}^{r} s^{2 l+2} g_{\Phi, \gamma}(s, \nu(s), \lambda(s), \varepsilon(s)) d s .
$$


Then we have the following inequality:

$$
B \leq C \gamma_{0} R^{4 l+2}+C \gamma_{0}^{4} R^{2 l+2},
$$

and we choose $\gamma_{0}$ sufficiently small to obtain

$$
1-B \geq 1-C \gamma_{0} R^{4 l+2}-C \gamma_{0}^{4} R^{2 l+2}>0 .
$$

Thus, we deduce the estimate for $I_{1}$ and $I_{2}$ :

$$
I_{1}, I_{2} \leq C \gamma, \gamma \in\left[0, \gamma_{0}\right]
$$

We now investigate an estimate for $I_{3}$. To do so, an estimate for the difference $\left|g_{\Phi, \gamma}(r, \nu(r), \lambda(r), \varepsilon(r))-g_{0}(U(r)+j(r))\right|$ is necessary. In what follows we are going to assume for simplicity that $\nu<0$. Thus

$$
\frac{e^{\gamma \nu(r)}-1}{\gamma} \geq \nu(r)
$$

and then

$$
t:=\frac{e^{\gamma \nu(r)}-1}{\gamma}+j(r) \geq \nu(r)+j(r)
$$

and this allows us to write

$$
\left|g_{\Phi, \gamma}(r, \nu(r), \lambda(r), \varepsilon(r))-g_{0}(U(r)+j(r))\right| \leq C\left(J_{1}+J_{2}+J_{3}\right)+C \gamma e^{2 \lambda(r)} \varepsilon^{2}(r),
$$

where

$$
\begin{aligned}
J_{1} & :=\left|e^{-\gamma(2 l+4) \nu(r)}-1\right| \int_{t}^{+\infty} \Phi(E)\left(\gamma E-\gamma t+e^{\gamma \nu}\right)^{2}\left[\frac{1}{\gamma}\left(\gamma E-\gamma t+e^{\gamma \nu}\right)^{2}-\frac{1}{\gamma} e^{2 \gamma \nu}\right]^{l+1 / 2} d E, \\
J_{2} & :=\int_{\nu(r)+j(r)}^{t} \Phi(E)(2 E-2 t)^{l+1 / 2} d E \\
J_{3} & :=\int_{t}^{+\infty} \Phi(E)\left|\left(\gamma E-\gamma t+e^{\gamma \nu}\right)^{2}\left[\frac{1}{\gamma}\left(\gamma E-\gamma t+e^{\gamma \nu}\right)^{2}-\frac{1}{\gamma} e^{2 \gamma \nu}\right]^{l+1 / 2}-(2 E-2 t)^{l+1 / 2}\right| d E .
\end{aligned}
$$

Next using the compactness of support of $\Phi$, one deduces that

$$
J_{1}, J_{2} \leq C \gamma .
$$

In order to obtain an estimate for $J_{3}$, one has

$$
\begin{aligned}
D & :=\left|\left(\gamma E-\gamma t+e^{\gamma \nu}\right)^{2}\left[\frac{1}{\gamma}\left(\gamma E-\gamma t+e^{\gamma \nu}\right)^{2}-\frac{1}{\gamma} e^{2 \gamma \nu}\right]^{l+1 / 2}-(2 E-2 t)^{l+1 / 2}\right| \\
& \leq C\left|\left(\gamma E-\gamma t+e^{\gamma \nu}\right)^{2}-1\right|+\left|\left(\frac{1}{\gamma}\left(\gamma E-\gamma t+e^{\gamma \nu}\right)^{2}-\frac{1}{\gamma} e^{2 \gamma \nu}\right)^{l+1 / 2}-(2 E-2 t)^{l+1 / 2}\right| \\
& \leq C \gamma+C\left|\frac{1}{\gamma}\left(\gamma E-\gamma t+e^{\gamma \nu}\right)^{2}-\frac{1}{\gamma} e^{2 \gamma \nu}-(2 E-2 t)\right|^{l+1 / 2} \\
& \leq C \gamma+C\left|\frac{1}{\gamma}\left(\gamma E-\gamma t+e^{\gamma \nu}\right)^{2}-\frac{1}{\gamma} e^{2 \gamma \nu}\right| \\
& \leq C \gamma+C \gamma^{\min (l+1 / 2,1)} .
\end{aligned}
$$


Besides, we observe that as in Lemma 3.1, $g_{0} \in C^{1}(\mathbb{R})$, and for all $t \geq \nu_{0},\left|g_{0}^{\prime}(t)\right| \leq C$. Thus

$$
I_{4} \leq \frac{C}{r^{2}} \int_{0}^{r} s^{2 l+2}\left|(\nu(s)-U(s))+\left(j(s)-j_{N}(s)\right)\right| d s,
$$

and we can combine all the above estimates to obtain

$$
\left|\nu^{\prime}(r)-U^{\prime}(r)\right| \leq C \gamma^{\min (l+1 / 2,1)}+\frac{C}{r^{2}} \int_{0}^{r} s^{2 l+2}\left(|\nu(s)-U(s)|+\left|j(s)-j_{N}(s)\right|\right) d s .
$$

So, (4.18) shows that an estimate for $j(r)-j_{N}(r)$ is needed. Using (4.11), (4.14), and (4.17), we have, because $\nu<0$,

$$
\begin{aligned}
\left|j^{\prime}(r)-j_{N}^{\prime}(r)\right| & =|q|\left|\left(e^{\gamma \nu+\lambda}-1\right) e^{\lambda} \varepsilon+\left(e^{\lambda} \varepsilon-\varepsilon_{N}\right)\right| \\
& \leq C \gamma+\left|e^{\lambda} \varepsilon-\varepsilon_{N}\right| .
\end{aligned}
$$

To get an estimate for $e^{\lambda} \varepsilon-\varepsilon_{N}$, let us integrate (4.10) and (4.12) over [0,r] and use the same method when estimating $I_{3}$ to obtain finally that

$$
\left|j^{\prime}(r)-j_{N}^{\prime}(r)\right| \leq C \gamma^{\min (l+1 / 2,1)}+\frac{C}{r^{2}} \int_{0}^{r} s^{2 l+2}\left(|\nu(s)-U(s)|+\left|j(s)-j_{N}(s)\right|\right) d s .
$$

Thus, adding (4.18) and (4.20) yields

$$
\begin{aligned}
& \left|\nu^{\prime}(r)-U^{\prime}(r)\right|+\left|j^{\prime}(r)-j_{N}^{\prime}(r)\right| \leq C \gamma^{\min (l+1 / 2,1)} \\
& \quad+\frac{C}{r^{2}} \int_{0}^{r} s^{2 l+2}|\nu(s)-U(s)|+\left|j(s)-j_{N}(s)\right| d s,
\end{aligned}
$$

for $\gamma \in\left[0, \gamma_{0}\right]$ and $r \in[0, R]$. So, using the Gronwall lemma, one obtains the following estimates:

$$
|\nu(r)-U(r)|,\left|j(r)-j_{N}(r)\right|,\left|e^{\lambda}-1\right|,\left|\left(e^{\lambda} \varepsilon-\varepsilon_{N}\right)(r)\right| \leq C \gamma^{\min (l+1 / 2,1)},
$$

and because

$$
\varepsilon-\varepsilon_{N}=\left(e^{\lambda} \varepsilon-\varepsilon_{N}\right)-\left(e^{\lambda}-1\right) \varepsilon,
$$

one deduces the same estimate for the left hand side of (4.21), completing the proof of Theorem 4.1.

We now make a comment on how to deduce from Theorem 4.1 that our solution is with finite radius and finite total mass, so that the corresponding spacetime is asymptotically flat. First of all, it is well known that a solution of the VP system has a finite radius and a finite total mass once one considers as in [14] the following ansatz for the distribution function $f$ :

$$
\Phi(E, L):=(-E)_{+}^{k} L^{l}, E \in \mathbb{R}, L>0,
$$

where $k \leq 0, l>-1 / 2, k<3 l+7 / 2$, and $(.)_{+}$is the positive part. In this context there exists $R>0$ such that the Newtonian potential $U$ satisfies $U(R)>0$. Besides, for $\gamma$ small, Theorem 4.1 tells us that $\nu(R)>0$ and in addition if $\nu_{0}<0$, we can use Theorem 4.2 of [14] to conclude that our solution $(\nu, \lambda, \varepsilon)$ makes the ADM mass

$$
M_{\infty}:=\lim _{r \rightarrow+\infty} m(r), \quad m(r)=4 \pi \int_{0}^{r} s^{2} \rho(s) d s
$$

be finite and then our obtained spacetime is asymptotically flat as announced before. 


\section{Conclusion}

In this work we planned to look for a global asymptotically flat static solution for the spherically symmetric EVM system, in the isotropic or anisotropic case. To achieve this, a first step consisted of extending the local solution obtained in Theorem 3.2 to a global one, using one generalized form of Gronwall's lemma. As a second step, Theorem 4.1 is crucial to obtain the finiteness of ADM mass and, having this in hand, one can deduce that our obtained spacetime is asymptotically Minkowskian in space as announced. One can also prove that this spacetime is geodesically complete.

\section{REFERENCES}

[1] A. Schulze, Existence and Stability of Static Shells for the Vlasov-Poisson System with Fixed Central Point Mass, Mathematical Proceedings of the Cambridge University press, 2009.

[2] A. Parry, Spherically symmetric static states of Wave Dark Matter, arXiv:1212.6426, 2012.

[3] H. Andréasson, M. Eklund, and G. Rein, A numerical investigation of the steady states of the spherically symmetric Einstein-Vlasov-Maxwell system, Class. Quantum Grav., 26(14), 145003, 2009.

[4] J. Batt and K. Pfaffelmoser, On the radius continuity of the models of polytropic gas spheres which correspond to the positive solutions of the generalized Emden-Fowler equation, Math. Meth. Appl. Sci., 10, 499-516, 1988.

[5] J. Batt, H. Berestycki, P. Degond, and B. Perthame, Some families of solutions of the VlasovPoisson system, Arch. Rat. Mech. Anal., 104, 79-103, 1988.

[6] J. Batt, W. Faltenbacher, and E. Horst, Stationary spherically symmetric models in stellar dynamics, Arch. Rat. Mech. Anal., 93, 159-183, 1986.

[7] J. Binney and S. Tremaine, Galactic Dynamics, Princeton University Press, Princeton, 1987.

[8] L. Perko, Differential Equations and Dynamical Systems, Springer-Verlag, New-York, 2001.

[9] M.E. Taylor, Partial Differential Equations, Basic Theory, Springer-Verlag, New-York, 115, 2001.

[10] P. Noundjeu, Global static solutions of the spherically symmetric Vlasov-Einstein-Maxwell (VEM) system for low charge, Adv. Pure Math., 3, 121-126, 2013.

[11] P. Noundjeu, The Einstein-Vlasov-Maxwell System with Spherical Symmetry, PhD thesis, Technical University of Berlin, April, 2005.

[12] P. Noundjeu and E. Ngondiep, On the non-relativistic limit of the spherically symmetric Einstein-Vlasov-Maxwell system, Annales de la Faculté des Sciences Université de Yaoundé, 37(1), 1-15, 2010.

[13] G. Rein and A.D. Rendall, Commpact support of spherically symmetric equilibria in nonrelativistic and relativistic galactic dynamics, Mathematical Proceedings of the Cambridge Philosophical Society, 128(02), 363-380, 2000.

[14] G. Rein, Static solutions of the spherically symmetric Vlasov-Einstein system, Mathematical Proceedings of the Cambridge Philosophical Society, 115(3), 559-570, 1994.

[15] G. Rein and A.D. Rendall, The Newtonian limit of the spherically symmetric Vlasov-Einstein system, Commun. Math. Phys., 150, 585-591, 1992.

[16] A.D. Rendall, Partial Differential Equations in General Relativity, Oxford University Press, 2008.

[17] S.L. Shapiro and S.A. Teukolsky, Relativistic stellar dynamics on the computer, II Physical applications, Astrophysical J., 298, 58-79, 1985.

[18] J. Smoller, A. Wassermann, S.T. Yau, and J. McLeod, Smooth static solutions of the EinsteinYang-Mills equations, Commun. Math. Phys., 143, 115-147, 1991. 